\title{
Mid-Infrared Cross-Comb Spectroscopy using Sum-Frequency Sampling
}

\author{
Mingchen Liu', Robert M. Gray ${ }^{1}$, Arkadev Roy ${ }^{1}$, Alireza Marandi ${ }^{1, ~ * ~}$ \\ ${ }^{1}$ Department of Electrical Engineering, California Institute of Technology, Pasadena, California, 91125, USA \\ "marandi@caltech.edu
}

\begin{abstract}
We introduce the cross-comb spectroscopy scheme by sum-frequency sampling of a mid-infrared frequency comb using a near-IR comb with slightly shifted repetition rate. We experimentally demonstrate a 300-nm instantaneous comb-resolved measurement bandwidth around $4 \mu \mathrm{m}$. (c) 2021 The Author(s)
\end{abstract}

Dual-comb spectroscopy (DCS), based on two mutually locked frequency comb sources in the same wavelength range, has been a compelling alternative to Fourier transform spectroscopy (FTIR) with numerous advantages [1]. Significant efforts have gone into the extension of this technology to the Mid-infrared (MIR) wavelength region $(2-20 \mu \mathrm{m})$, where molecular signatures are strong and ubiquitous. However, making two mutually locked broadband frequency comb sources in the mid-IR have been a significant challenge. Moreover, photodetectors in the MIR usually suffer from lower sensitivity, higher noise, and slower response time compared to their well-developed near-IR (NIR) counterparts. One effective way to overcome these limitations is to upconvert MIR frequency combs (FCs) to the NIR and capture the wealth of molecular information using another NIR comb with NIR detectors. One recent successful example of this approach is asynchronous electro-optic sampling (A-EOS) in which ultrashort NIR pulses with slightly detuned repetition rate are used to directly detect the electric field of MIR pulses in the time domain [2,3]. However, this method necessitates extremely short NIR pulses with duration shorter than the optical cycle of the carrier frequency of the MIR pulses [4,5], whose generation and dispersion control require substantial efforts. Moreover, the detection is based on field-dependent polarization rotation of NIR sampling pulses, which adds complexity to the system. In addition to A-EOS, one can also up-convert the MIR frequency comb using a high power NIR c.w. laser and perform a standard DCS in the NIR, as demonstrated lately [6]. Owing to the low up-conversion efficiency, this method doesn't usually have favorable SNR and bandwidth compared to direct MIR DCS.

Here we introduce a new method named Cross-Comb Spectroscopy (CCS). As illustrated in Fig. 1, the spectral information contained in the MIR (target) FC of interest is upconverted to the NIR region via sum-frequency-generation (SFG) with a NIR (local) FC of a slightly shifted repetition rate (by $\delta$ ). The SFG output is then interfered by the spectral extension of the local FC (readout FC). Similar to DCS, it is possible to one-to-one map the MIR FC teeth to the radio frequency (RF) comb teeth that are easily accessible with single NIR detector and RF measurement. We demonstrate that, to obtain the teeth-resolved absorption spectrum of the target $\mathrm{FC}$, the minimal required total bandwidth of the local and readout $\mathrm{FC}$ is about equal to the bandwidth of the target FC, which eliminates the need for super short NIR pulse generation and measurement of polarization rotation compared to A-EOS.

(a)

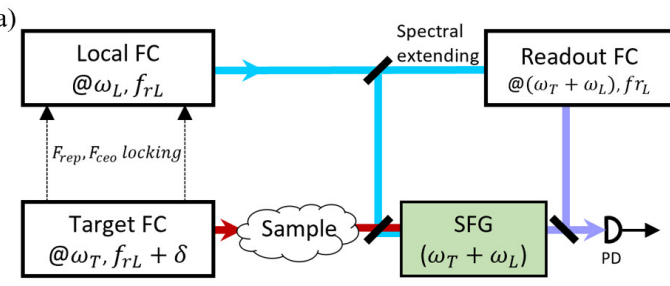

(b)

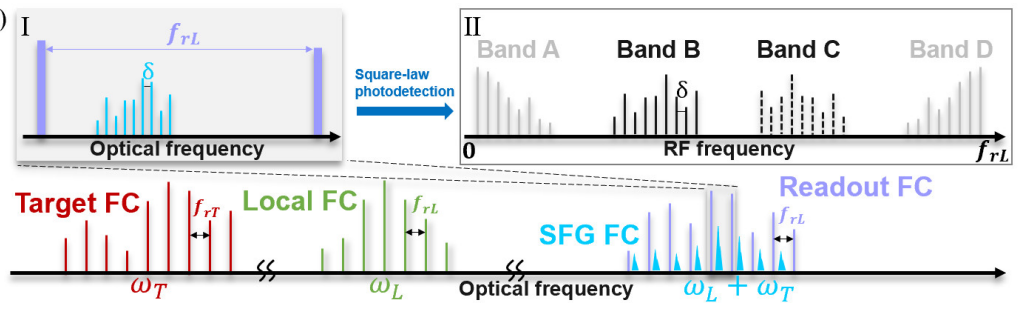

Fig. 1 (a) Schematic of CCS. (b) Principle of CCS in the frequency domain. Inset I illustrates one group of SFG FC teeth and its nearest readout teeth, and inset II illustrates RF FC obtained by square-law detection of the heterodyne beating between SFG FC and readout FC with a single NIR detector.

Fig 1(b) illustrate the principle of the method. Each pair of comb teeth from the local (green) and target FC (red) will generate an SFG tooth at a unique frequency, the set of which are referred to as SFG FC (blue). The teeth of SFG FC cluster into different frequency groups, which are evenly spaced by $f_{r L}$ [7]. There are some patterns of the distribution of the SFG FC 
within one group, that teeth are separated by $\delta$ (inset I), and the teeth generated by a certain target tooth are always placed at a unique and unchanged relative position, which makes it possible to do a one-to-one mapping from target teeth to RF. To realize this, a readout FC (purple), which is effectively a spectral extension of the local FC and thereby share the same $f_{r}$ and $f_{c e o}$, is employed here to beat with the SFG FC on a NIR photodetector. The resultant RF FC can be distinguished to 4 bands (inset II). While band $\mathrm{A}$ and $\mathrm{D}$ are complicated mixtures that are challenging to decipher, band $\mathrm{B}$ (or its mirror image band $\mathrm{C}$ ) is simply a one-to-one mapping from target FC (multiplied by the local and readout FCs) to RF FC.
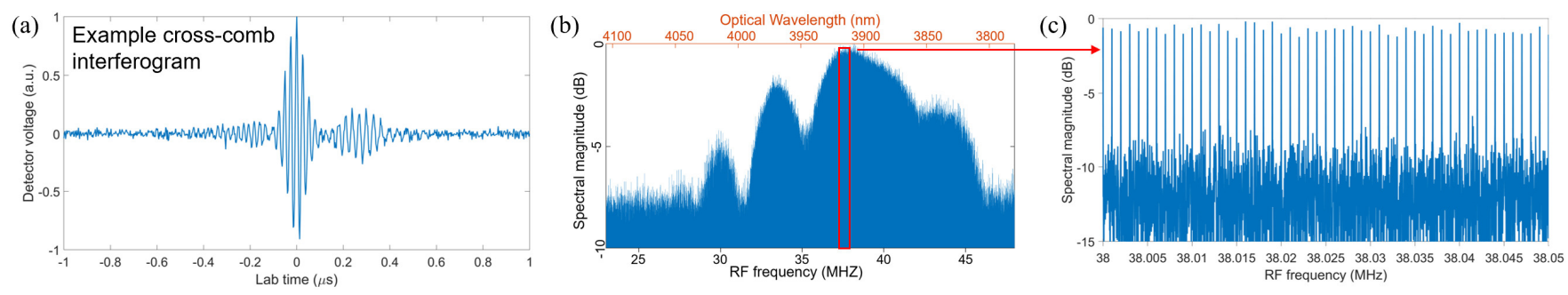

Fig. 2 Experimental results of CCS at around $4 \mu \mathrm{m}$. (a) A typical interferogram obtained in the experiment, zoomed in to show the center-burst. The repetition period of interferograms is $1 \mathrm{~ms}$, corresponding to $\delta=1 \mathrm{kHZ}$. (b) Spectral magnitude of Bands B of RF FC obtained by averaging of 50 interferograms ( 50 ms averaging time). (c) a zoomed-in view of (b), which manifests the resolved comb lines with a spacing equal to $\delta$.

Thanks to the one-to-one teeth mapping, to interrogate the sample response within the target comb, one just need to normalize the measured sample-in band B (or C) with the sample-free result. It can be shown that, in theory, the one-to-one mapping requires $(L+R) \geq(T+1)$, where $L, R$ and $T$ denote the number of comb teeth of local, readout and target FC, respectively. Provided that one-to-one mapping is fulfilled, the maxima optical spectral bandwidth for target combs is $f_{r L}^{2} / 2 \delta$ or $f_{r L}^{2} / 4 \delta$ depends on if a balance detection is used or not.

Our experimental results of MIR cross-comb spectroscopy around $4 \mu \mathrm{m}$ are shown in Fig. 2. The target FC is a femtosecond frequency comb centered at $4.2 \mu \mathrm{m}$ of a 50 -fs pulse width, $500-\mathrm{mW}$ average power and $250-\mathrm{MHZ}$ repetition rate, generated from a chain of two cascaded half-harmonic OPOs [8] which is intrinsically locked to the pump frequency comb at 1 um. The local FC is a NIR FC centered at $1560 \mathrm{~nm}$ with a $\sim 15-\mathrm{nm}$ FWHM bandwidth and $\sim 80 \mathrm{~mW}$ of average power, and the readout FC is a band-pass-filtered part of supercontinuum pumped by the local FC, with a 10-dB spectral coverage from $1100 \mathrm{~nm}$ to $1150 \mathrm{~nm}$. The $f_{\text {rep }}$ of the target $\mathrm{FC}$ is locked to that of the local FC with a $\delta=1 \mathrm{kHZ}$, which is locked against a RF Rb standard. Cross-comb spectroscopy is achieved by SFG from the MIR target FC and the local FC (i.e. the sum-frequency sampling of the MIR comb) in a 1-mm-long periodically poled lithium niobate (PPLN) crystal followed by its interference with the readout FC, which is measured by an InGaAs balanced detector. The PPLN crystal has a $29.08-\mu \mathrm{m}$ poling period which can provide a $\sim 400$-nm quasi-phase-matching bandwidth for the SFG. As shown in Fig. 2(b), $2.5 \times 10^{4}$ comb lines corresponding to the 4$\mu \mathrm{m}$ target spectrum multiplied by the local and readout FC are measured, which cover a 6.25 -THZ $\operatorname{spectrum}(3.8 \mu \mathrm{m}-4.1 \mu \mathrm{m})$ for the target FC. The upper axis of the plot shows a rough optical wavelength calibration for target.

It is worth noting that the current results are obtained by only locking the $f_{\text {rep }}$ of the target and local combs while their relative $f_{c e o}$ is free running. After locking these CEO frequencies, we will be able to accurately calibrate the optical frequencies of the comb lines and achieve MIR frequency comb spectroscopy of several molecular species. We expect to be able to extend the instantaneous spectral coverage of the measurement by using a shorter PPLN crystal, and improve the SNR by longer averaging, more stable frequency locking, and proper phase correction algorithms.

In summary, we propose and experimentally demonstrate the concept of cross-comb spectroscopy in the MIR using sumfrequency sampling of a 4 -um comb by a 1.5 -um comb. Cross-comb spectroscopy combines the merits of DCS and A-EOS while circumventing some of their practical challenges.

\section{Reference}

1. I. Coddington et al., Optica, 3(4), 414-426 (2016).

2. A. S. Kowligy et al., Science Advances 5(6), 8794 (2019).

3. I. Pupeza et al., Nature 577(7788), 52-59 (2020).

4. R. A. Stead et al., J. Opt. Soc. Am. B, 29(10), 2861-2868 (2012).

5. C. Riek et al., Science 350(6259), 420-423 (2015).

6. Z. Chen et al., arXiv:2003.06930 [physics] (2020).

7. S. Yokoyama et al., Opt. Express, 17(20), 17324-17337 (2009).

8. E. Sorokin et al., Opt. Express, 26(8), 9963-9971 (2018) 